\title{
Stability of Fluctuations in Alkali-Cellulose Concentration in Continuous Steeping, Pressing and Disintegration Process
}

\author{
By Kumeo Nakajima, Member, TMSJ \\ Faculty of Engineering, University of Osaka Prefecture, Osaka Pref. \\ Based on Journal of the Textile Machinery Society of Japan, 17, T717-724(1964)
}

\begin{abstract}
In the previous paper, the behavior of a slurry tank was expressed by a difference differential equation. Thus, the instability of the oscillation of the concentration of the slurry delivered from the tank was explained.

In the present paper, the author discusses how to make the concentration oscillation stable. It would be achieved by introducing a servo-mechanism feeding the slurry concentration back to the pulp supply rate. Unfortunately, the continuous measurement of the slurry concentration is very difficult, if not impossible.

In order to avoid this difficulty, the author has analyzed the dynamic performance of the whole system from the slurry tank to the slurry press. It has revealed that the pressure of the surge tank depends upon the concentration of the slurry delivered from the slurry tank. The pressure of the surge tank is easily detected continuously. The rate of the pulp supply to the slurry tank is regulated through a servo-mechanism actuated by the pressure of the surge tank.

The characteristic equation of the whole system, including the servo, is derived and transformed into a form suitable for applying the Satche's method. Hence, the whole system can easily be stabilized, even if the pure delay is longer than the transportation -lag of the slurry tank.

The feedback control not only stabilizes the system, but also ameliorates the fre. quency response of the whole system.
\end{abstract}

\section{Introduction}

The previous paper[1] suggested a mathematical model of a slurry tank composed of a transportation lag and a pure delay. The paper explained the instability of concentration fluctuation of alkali-cellulose delivered from a slurry tank when the production rate was increased beyond a certain limit.

A question arises whether the instability is unavoidable or not. The present paper investigates theoretically into this problem and discloses that it is possible to make the whole process stable by adding a feedback control loop.

\section{Nomenclature}

In this paper the following nomenclature is used, functions in time domain being expressed as a sum of a steady value and a deviation therefrom:

$V_{0}$ : Volume of slurry tank, including circulation pipings

$\tau_{1} \quad$ : Pure delay in slurry tank

$q_{1}$ : Volumetric rate of flow of slurry discharged from slurry tank and fed to surge tank

$A$ : Sectional area of surge tank

$H$ : Height of surge tank

$\gamma$ : Average weight of slurry per unit volume in surge tank

$\tau_{2} \quad$ : Pure delay in surge tank

$R \quad$ : Piping resistance of exit side of surge tank

$V_{4}$ : Volume of reservoir of slurry press

$q_{5}$ : Volumetric rate of flow of slab discharged from slurry press

$k \quad$ : Constant of proportionality

$t \quad:$ Time

$s$ : Auxiliary variable of Laplace transform

$F(s)$ : Transfer function of servo-mechanism

$G(s)$ : Transfer function to be compared

$\omega$ : Frequency

$u$ : Non-dimensional frequency

$K$ : Gain

$T$ : Time constant

$\zeta \quad$ : Damping ratio

$\alpha \quad$ : Rate of transportation lag against pure delay $\tilde{q}_{0}(t)=\bar{i}_{0}+q_{0}(t)$ : Equivalent volumetric rate of feed- 
ing of alkali-cellulose into slurry tank

$\tilde{r}(t)=\bar{r}+r(t)$ : Reference value of the system

$\tilde{c}_{0}(t)=\bar{c}_{0}+c_{0}(t)$ : Average equivalent volumetric concentration of alkali-cellulose in slurry tank (space average at each instant)

$\widetilde{c}_{1}(t)=\bar{c}_{1}+c_{1}(t)$ : Volumetric concentration of alkalicellulose in slurry fed to surge tank

$\tilde{x}(t)=\bar{x}+x(t)$ : Height of air space in surge tank

$\tilde{p}_{2}(t)=\bar{p}_{2}+p_{2}(t)$ : Air pressure in surge tank

$\tilde{c}_{2}(t)=\bar{c}_{2}+c_{2}(t)$ : Average volumetric concentration of alkali-cellulose in surge tank (space average at each instant)

$\tilde{q}_{3}(t)=\bar{q}_{3}+q_{3}(t)$ : Volumetric rate of flow of slurry discharged from surge tank and fed to slurry press

$\tilde{c}_{3}(t)=\bar{c}_{3}+c_{3}(t)$ : Volumetric concentration of alkalicellulose in slurry fed to slurry press

$\tilde{p}_{4}(t)=\bar{p}_{4}+p_{4}(t)$ : Pressure of slurry in reservoir of slurry press

$\tilde{q}_{4}(t)=\bar{q}_{4}+q_{4}(t)$ : Volumetric rate of flow of caustic soda solution discharged from slurry press

$\tilde{c}_{4}(t)=\bar{c}_{4}+c_{4}(t)$ : Average volumetric concentration of alkali-cellulose in slurry in reservoir of slurry press (space average at each instant)

$\tilde{c}_{5}(t)=\bar{c}_{5}+c_{5}(t)$ : Volumetric concentration of alkalicellulose in slurry discharged from slurry press

The motion of equivalent rate of flow and concentration of alkali-cellulose is introduced to avoid the complexity of volume change due to formation of alkali-cellulose. Actually, pulp and caustic soda solution is fed into the slurry tank. A reaction of cellulose to caustic soda takes place. Here it is assumed that alkalicellulose is proportional in volume to cellulose before the reaction takes place. Thus the equivalent rate of flow and concentration is derived by converting the volume of cellulose into that of alkali-cellulose.

\section{Assumptions}

The following assumptions are used to derive the fundamental equations of the whole process :

(1) A slurry tank is expressed by a mathematical model composed of a transportation lag and a pure delay.

(2) The rate of pulp-feeding is continuous.

(3) No cellulose is contained in the overflow from the slurry tank.

(4) The alkali-cellulose is proportional in volume to cellulose before the reaction takes place.

(5) The rate of flow of slurry discharged from the slurry tank and fed to the surge tank is kept constant.
(6) A surge tank is expressed by a mathematical model composed of a transportation lag and a pure delay. However, the pure delay of the surge tank is much smaller than the pure delay of the slurry tank and the time constant of the surge tank. Hence the pure delay of the surge tank is ignored.

(7) The weight of slurry per unit volume in the surge tank is constant.

(8) The pressure-volume relationship of the air is expressed by Boyle's law.

(9) The slurry press separates the slab and the caustic soda solution from the slurry. The slab discharged from the slurry press is assumed to be a continuous flow as if it were a fluid with the notions of rate of flow and concentration of alkali-cellulose applicable.

(10) The rate of flow of the caustic soda solution separated by, and discharged from the slurry press, is proportional to the pressure of the slurry in the reservoir of the press, and is inversely proportional to the concentration of the alkali-cellulose of the slurry.

(11) The caustic soda solution mentioned above contains no alkali-cellulose.

(12) The rate of flow of the slab discharged from the slurry press is a constant determined by the clearance and the surface speed of cage rollers.

(13) The concentration of alkali-cellulose in the slab is proportional to that of the slurry in the reservoir of the slurry press.

(14) The rate of flow and the concentration are all expressed by volume; and chemical reaction causes no change in the volume of slurry after it leaves the slurry tank.

(15) The transportation lag in the pipings is negligible.

(16) Slurry is incompressible.

(17) Deviations of variables from their steadystate values are small and their higher-order terms negligible.

(18) Thermal effects are ignored.

These assumptions include some bold ones, particularly those concerning the slurry press. However, the object of the present paper is not to analyse the behavior of each element but to discuss the possibility of stabilizing the whole process. Hence a more detailed analysis of each element is omitted.

\section{Stabilization of Slurry Tank}

Fundamental equations of the slurry tank are given by the following equations derived from the discussion 
in the previous paper

$$
\begin{aligned}
& V_{0}(d / d t) \tilde{c}_{0}(t)=\tilde{q}_{0}(t)-q_{1} \cdot \tilde{c}_{1}(t), \\
& \tilde{c}_{0}(t)=\tilde{c}_{1}\left(t+\tau_{1}\right),
\end{aligned}
$$

the variables being the sums of the steady-state value and the deviations therefrom. Thus, the following equations are delivered:

$$
\begin{aligned}
& \bar{q}_{0}=q_{1} \cdot \bar{c}_{1}, \\
& \bar{c}_{0}=\bar{c}_{1}, \\
& V_{0}(d / d t) c_{0}(t)+q_{1} \cdot c_{0}(t-\tau)=q_{0}(t),
\end{aligned}
$$

The previous paper discussed the stability of eq. 5 analytically. For the purpose of the present paper it is more convenient to use a graphical method, originally suggested by $M$. Satche[2] and ingeneously applied by H.S. Tsien to the stability problem of the rocket motor with liquid propellant.[3]

Take the Laplace transform of both sides of eq. 5 ,

$$
\begin{aligned}
C_{0}(s) / Q_{0}(s)= & 1 /\left(V_{0} s+q_{1} e^{-\tau_{1} s}\right) \\
& =K_{1}^{\prime} /\left(T_{1}^{\prime} s+e^{-\tau_{1}}\right),
\end{aligned}
$$

where

$$
K_{1}{ }^{\prime}=1 / q_{1}, \quad T_{1}{ }^{\prime}=V_{0} / q_{1},
$$

Then the characteristic equation of the slurrry tank is expressed by

$$
T_{1}^{\prime} s+e^{-\tau_{1}}{ }^{s}=0 .
$$

The question of whether any of the characteristic roots has a positive real part is solved by Nyquist's method. Satche's method is an improvement on Nyquist's for systems having a pure delay.

Assuming, in this case :

$$
g_{1}(s)=e^{-\tau_{1} s}, g_{2}(s)=-T_{1}{ }^{\prime} s,
$$

eq. 8 is written as

$$
G(s)=g_{1}(s)-g_{2}(s)=0 .
$$

Polar plots of $g_{1}(s)$ and $g_{2}(s)$ and the correspondence of $g(s)$ vectors to $s$-vector is illustrated in Fig. 1.

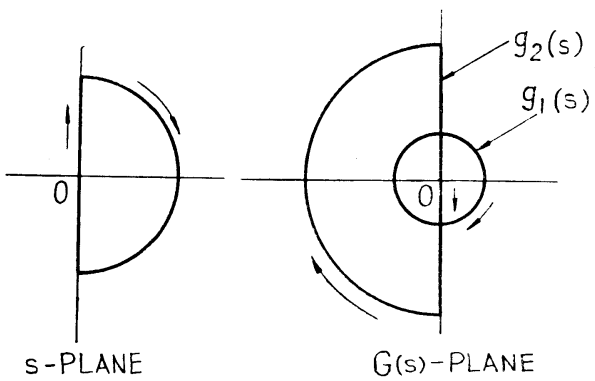

Fig. 1 Correspondence of $g(s)$-plots to s-plot

As shown in Fig. 1, $g_{2}$-plot crosses $g_{1}$-plot. Satche's criterion tells us that the system is stable when $\tau_{1}<(\pi / 2) K_{1}^{\prime}$.

This is the same condition as that derived analytically in the previous paper.

This inequality is no longer satisfied if the production rate is increased excessively. To avoid trouble, it is necessary to make the system absolutely stable.
The polar plot $g_{2}(s)$ should be brought to the outside the polar plot $g_{1}(s)$ to - the left of $g_{1}(s)$.

This is achieved by introducing a servo-mechanism such as is shown in Fig. 2. Its block diagram is illustrated in Fig. 3. The transfer function of the closed system is

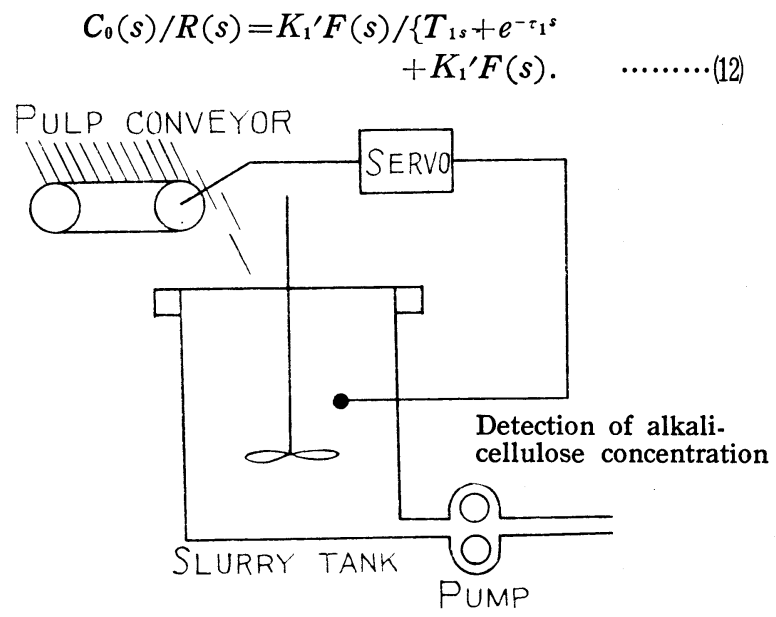

Fig. 2 Feedback control of slurry tank

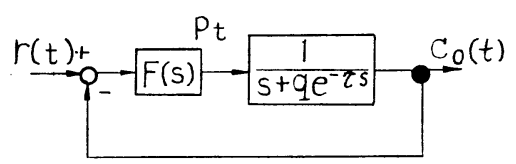

Fig. 3 Block diagram of feedback control of slurry tank

The characteristic equation is

$$
e^{-\tau_{1} s}+\left\{T_{1 s}^{\prime}+K_{1}^{\prime} F(s)\right\}=0,
$$

and instead of eq. 9

$$
g_{1}(s)=e^{-\tau_{1} s}, g_{2}(s)=-\left\{T^{\prime}{ }_{1 s}+K_{1}^{\prime} F(s)\right\} . \quad \cdots(14)
$$

$F(s)$ here should be so selected as to keep the $g_{2}$-plot to the left of $g_{1}$-plot.

If $F(s)$ is selected as

$$
F(s)=K, K K_{1^{\prime}}>1 \text {, }
$$

$g_{2}$-plot gets completely outside $g_{1}$-plot, as shown in Fig. 4. This means that the system is absolutely stable for any value of $\tau_{1} / K_{1^{\prime}}$.

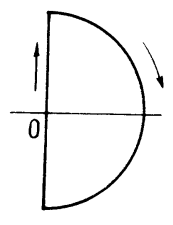

S-plane

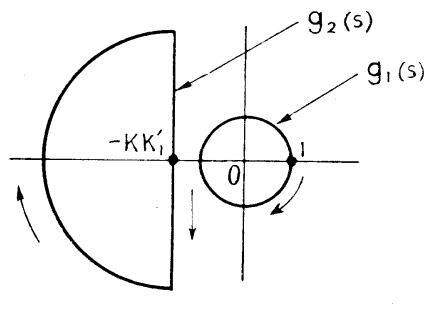

G(s)-plane
Fig. 4 Correspondence of $g(s)$-plots to s-plot

Servo motors usually have a transfer function of the type: 


$$
F(s)=K /(1+T s) .
$$

If $K K_{1}^{\prime}$ in this case exceeds one, $g_{2}$-plot again gets completely outside $g_{1}$-plot, making the system absolutely stable.

In practice, this method has two major drawbacks. First, the detection of average value $c_{0}(t)$ is very difficult. Second, there is no good instrument available in measuring the alkali cellulose concentration in the slurry continuously. Other means should, therefore, be developed. One conceivable means is to study the behavior of the subsequent stages of the whole process and obtain a measurable quantity which could be satisfactorily fed back to the feeding rate of cellulose.

\section{Basic Equations of Whole Process}

A sketch of the whole process is shown diagramatically in Fig. 5. Let us derive the basic equations of the whole process by using the assumptions listed above.

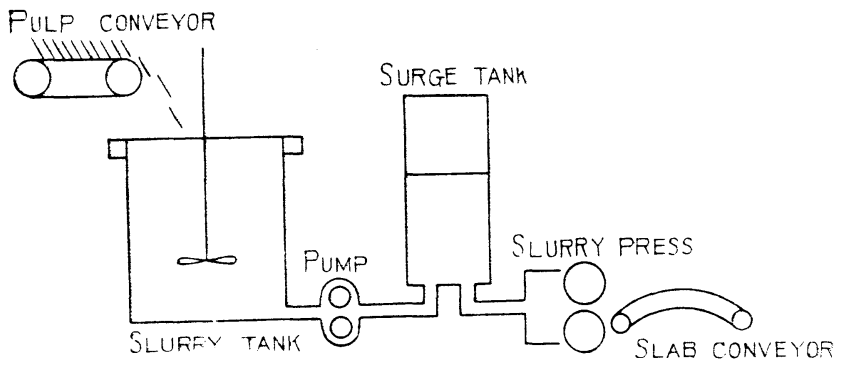

Fig. 5 Sketch of whole process

As is described above the slurry tank is assumed to have the basic equations given by eqs. 1 and 2 .

Those of the surge tank are expressed thus:

$$
\begin{aligned}
& A(d / d t) \widetilde{x}(t)=\tilde{q}_{3}(t)-q_{1}, \\
& A(d / d t)\left[\{H-\tilde{x}(t)\} \bar{c}_{2}(t)\right] \\
& =q_{1} \cdot \widetilde{c}_{1}(t)-q_{3} \cdot \tilde{c}_{3}(t), \\
& A \cdot \tilde{T}_{2}(t) \cdot \widetilde{x}(t)=k \text {, } \\
& \tilde{q}_{3}(t)=(1 / R)\left\{\tilde{f}_{2}(t)-\tilde{p}_{4}(t)\right\} \\
& +(\gamma / R)\{H-\tilde{x}(t)\} \\
& \bar{c}_{2}(t)={ }_{3}\left(t+\tau_{2}\right) .
\end{aligned}
$$

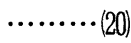

Basic equations of the slurry press are given by $\tilde{q}_{3}(t)=\tilde{q}_{4}(t)+\tilde{q}_{5}(t)$, $V_{4}(d / d t) \tilde{c}_{4}(t)=\tilde{q}_{3}(t) \cdot \tilde{c}_{3}(t)$

$$
-q_{5} \cdot \hat{c}_{5}(t)
$$

$\tilde{q}_{4}(t)=k_{5} \cdot \tilde{p}_{4}(t)+k_{6} / \check{\llcorner}_{4}(t)$,

.........(24)

$\tilde{C}_{5}(t)=k_{7} \cdot \tilde{c}_{4}(t)$.

Thus, 11 equations are obtained relative to 12 variables. It is possible to eliminate 10 variables from these equations and obtain a relation between the re. maining two. These two variables are so selected that $\tilde{q}_{0}(t)$ is input and $\hat{c}_{5}(t)$ output.
The 11 basic equations lead to the following further equations connecting the steady-state values of each variable :

$$
\begin{aligned}
& 0=\bar{q}_{3}-q_{1}, \\
& 0=q_{1} \cdot \bar{c}_{1}-\bar{q}_{3} \cdot \bar{c}_{3}, \\
& \bar{F}_{2} \cdot \bar{x}=k / A, \\
& \bar{q}_{2}=(1 / R)\left\{\left(\bar{p}_{2}-\bar{p}_{4}\right)+\gamma(H-\bar{x})\right\} \\
& \bar{c}_{2}=\overline{-}_{3}, \\
& \bar{q}_{3}=\bar{q}_{4}+q_{5}, \\
& 0=\bar{q}_{3} \cdot \bar{c}_{3}-q_{5} \cdot \bar{c}_{5} \\
& \bar{q}_{4}=k_{5} \cdot \bar{p}_{4}+k_{6} / \bar{c}_{4}, \\
& \bar{c}_{5}=k_{7} \cdot \bar{c}_{4} .
\end{aligned}
$$

Solving simultaneous equation 26 and eqs. 3 and 4 results in :

$$
\begin{aligned}
& \bar{c}_{0}=\bar{c}_{1}=\bar{c}_{2}=\bar{c}_{3}=\bar{q}_{0} / q_{1}, \\
& \bar{c}_{4}=\bar{q}_{0} / q_{5} k_{7}, \bar{c}_{5}=\bar{q}_{0} / q_{5}, \quad q_{1}=\bar{q}_{3}, \\
& \bar{q}_{4}=q_{1}-q_{5}, \quad \\
& \bar{F}_{2}=k / A \bar{x}, \bar{p}_{4}=\left(1 / k_{5}\right)\left\{\left(q_{1}-q_{5}\right)\right. \\
& \left.\quad-k_{6} k_{7} q_{5} / \bar{i}_{0}\right\}, \\
& \gamma \bar{x}^{2}+\left(q_{1} R+\bar{p}_{4}-\gamma H\right) \bar{x}-k / A=0,
\end{aligned}
$$

Thus $\bar{c}_{0}, \bar{c}_{1}, \bar{c}_{2}, \bar{c}_{3}, \bar{c}_{4}, \bar{c}_{5} ; \bar{q}_{3}, \bar{q}_{4} ; \bar{F}_{2}, \bar{p}_{4}$ and $\bar{x}$ are determined by only three constants $\bar{q}_{0}, q_{1}$ and $q_{5}$.

Equations of deviations to correspond to eq. 26 are given as follows:

$$
\begin{aligned}
& V_{0}(d / d t) c_{0}(t)=q_{0}(t)-q_{1} \cdot c_{1}(t), \\
& c_{0}(t)=c_{1}(t+\tau), \\
& A(d / d t) x(t)=q_{3}(t), \\
& A(d / d t)\left[\{H-x(t)\} \cdot \bar{c}_{2}(t)+(H-\bar{x})\right. \\
& \left.\quad \cdot c_{2}(t)\right]=\bar{q}_{1} \cdot c_{1}(t)-\bar{q}_{3} \cdot c_{3}(t)-\bar{c}_{3} \cdot q_{3}(t), \\
& 0=\bar{p}_{2} \cdot x(t)+\bar{x} \cdot p_{2}(t), \\
& q_{3}(t)=(1 / R)\left\{p_{2}(t)-p_{4}(t)-\gamma \cdot x(t)\right\}, \\
& c_{2}(t)=c_{3}(t+\tau), \\
& q_{3}(t)=q_{4}(t), \\
& V_{1}(d / d t) c_{4}(t)=\bar{q}_{3} \cdot c_{3}(t)+\bar{c}_{3} \cdot q_{3}(t) \\
& \quad-q_{5} \cdot c_{5}(t), \\
& q_{4}(t)=k_{5} \cdot p_{4}(t)-\left(k_{6} / \bar{c}_{4}{ }^{2}\right) \cdot c_{4}(t), \\
& c_{5}(t)=k_{7} \cdot c_{4}(t)
\end{aligned}
$$

Higher-order terms in these equations are ignored.

\section{Transfer Function of_Whole Process}

By taking the Laplace transform of eq. 28 and eliminating $C_{0}(s), C_{2}(s)$ and $X(s)$, the following equations are obtained:

$$
\begin{aligned}
& \left(V_{0} s e^{\tau_{1} s}+q_{1}\right) \cdot C_{1}(s)=Q_{0}(s), \\
& \{R+(\gamma / A)(1 / s)\} Q_{3}(s)=P_{2}(s)-P_{4}(s) \\
& \left(\bar{f}_{2} / A\right) \cdot Q_{3}(s)=-\bar{x} \cdot s P_{2}(s), \\
& \left\{A(H-\bar{x}) \cdot s e^{\tau_{2}} s+q_{1}\right\} \cdot C_{3}(s)=q_{1} \cdot C_{1}(s), \\
& Q_{3}(s)=k_{5} \cdot P_{4}(s)-\left(k_{6} / \bar{c}_{4}^{2}\right) \cdot C_{4}(s), \\
& \left(V_{4} s+q_{5} k_{7}\right) \cdot C_{4}(s)=q_{1} \cdot C_{3}(s) \\
& \quad+\left(\bar{q}_{0} / q_{1}\right) \cdot Q_{3}(s), \\
& C_{5}(s)=k_{7} \cdot C_{4}(s) .
\end{aligned}
$$




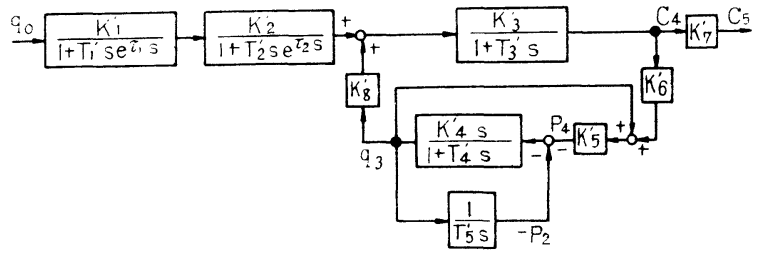

Fig. 6 Block diagram of whole process

A block diagram of eq. 29 is shown in Fig. 6. The gains and the time constants are written as:

$$
\left.\begin{array}{l}
K_{1}^{\prime}=1 / q_{1}, \quad K_{2}^{\prime}=q_{1}, \quad K_{3}^{\prime}=1 / q_{5} k_{7}, \\
K_{4}{ }^{\prime}=A / \gamma, \quad K_{5}^{\prime}=1 / k_{5}, \quad K_{6}{ }^{\prime}=k_{6} / \bar{c}_{4}{ }^{2} \\
K_{7^{\prime}}=k_{7}, \quad K_{8^{\prime}}=\bar{q}_{0} / q_{1}, \\
T_{1}{ }^{\prime}=V_{0} / q_{1}, T_{2}{ }^{\prime}=A(H-\bar{x}) / q_{1}, \\
T_{3}{ }^{\prime}=V_{4} / q_{5} k_{7}, \quad T_{4}^{\prime}=R A / \gamma, \quad T_{5}{ }^{\prime}=A \bar{x} / \bar{p}_{2} .
\end{array}\right\}
$$

From eq. 29 or Fig. 6 the transfer function of the whole process $C_{5}(s) / Q_{0}(s)$ is calculated.

$$
C_{5}(s)=\frac{K_{0}\left(1+T_{4} s\right)}{\left(1+T_{1} s e^{\tau_{1}} s\right)\left(1+T_{2} s e^{\tau_{2}} s\right)\left(1+2 \zeta T_{3} s+T_{3}^{2} s^{2}\right),}
$$

where

$$
\begin{aligned}
& K_{0}=1 / q_{5}, \quad T_{1}=V_{0} / q_{1}, T_{2}=A(H-\bar{x}) / q_{1}, \\
& T_{3}=\left\{V_{4} A \bar{x}\left(1+k_{5} R\right) / q_{5} k_{5} k_{7}\left(\bar{p}_{2}+\gamma \bar{x}\right)\right\}^{\frac{1}{2}}, \\
& T_{4}=A \bar{x}\left(1+k_{5} R\right) / k_{5}\left(\bar{E}_{2}+\gamma \bar{x}\right), \\
& \zeta=\left\{V_{4} k_{5}\left(\bar{E}_{2}+\gamma x\right)+q_{5} k_{7} A \bar{x}\left(1+k_{5} R\right)\right. \\
& \left.\quad+\bar{q}_{0} k_{6} A \bar{x} / q_{1} \bar{c}_{4}^{2}\right\} \cdot\left\{q_{5} k_{5} k_{7}\left(\bar{E}_{2}+\gamma x\right)\right\}^{\frac{1}{2}} \\
& \quad / 2\left\{V_{4} A \bar{x}\left(1+k_{5} R\right)\right\}^{\frac{1}{2}} .
\end{aligned}
$$

Additional transfer functions which will be used later are :

$$
\frac{C_{3}(s)}{Q_{0}(s)}=\frac{K_{1}^{\prime}}{\left(1+T_{1} s e^{\tau} 1^{s}\right)\left(1+T_{2} s e^{\tau} 2^{s}\right),}
$$

and

$$
\begin{aligned}
& C_{4}(s) \\
& P_{2}(s)
\end{aligned}=K_{1}\left(1+T_{4} s\right),
$$

where

$$
K_{1}=\left(k_{5} \bar{c}_{4}^{2} / k_{6}\right) \cdot\left(\bar{F}_{2}+\gamma \bar{x}\right) / \bar{I}_{2}
$$

\section{Discussion}

The first and second terms in the denominator of eq. 31 come from the slurry tank and the surge tank, respectively. Unless the inequalities

$\tau_{1}<(\pi / 2) T_{1}, \tau_{2}<(\pi / 2) T_{2}$ …...... 355

are satisfied, the whole process becomes unstable.

Even if the inequalities are satisfied, the gain plot of the frequency response exhibits marked peaks near $\omega=1 / T_{i}$ when $\tau_{i}$ is nearly equal to $(\pi / 2) T_{i}(i=1$ and 2$)$. The peak near $\omega=1 / T_{1}$ is more pronounced, because normally $\tau_{2} \ll \tau_{1}$ and $T_{2}<T_{1}$.

The gain of eq. 31 tends to $0 \mathrm{db}$ as $\omega$ tends to zero, decreases as $\omega$ increases. The whole process works as if it were a low-pass filter. Other than the gain peaks mentioned above, another peak may appear near $\omega=1 / T_{3}$ when $\zeta$ is small. If $T_{4}$ is small, the gain may become greater than $0 \mathrm{db}$ near $\omega=1 / T_{4}$.

Consider the function of the surge tank. Eq. 33 suggests that the surge tank cannot stabilize the process once resonance occurs in the slurry tank. In case resonance does not occur in the slurry tank, fluctuations in the alkali-cellulose concentration would be attenuated in a frequency range beyond $1 / T_{2}$, if $\tau_{2}$ were much smaller than $T_{2}$. However, $T_{2}$ is normally smaller than $T_{1}$ in actual tanks. Hence concentration fluctuations are not attenuated if frequency is below $1 / T_{2}$. Only higher frequency fluctuations exceeding $1 / T_{2}$ are attenuated. Thus, the effect of surge tank is not so marked in attenuating the concentration fluctuations.

What principal part does the surge tank play? Eq. 34 connects surge tank pressure $p_{2}(t)$ with alkali.cellulose concentration $c_{4}(t)$ in the slurry press. Too high an alkali-cellulose concentration in the slurry press may clog the slurry press; this is a fatal accident to a viscose mill. The surge tank should, therefore, be thought of as a safety device against clogging of the slurry press.

\section{Stabilization by Feedback Control}

As explained in Section 4, direct stabilization of slurry tank is practically impossible, because continuous measurement of $c_{0}(t)$ is difficult. However, the basic equations of the whole process derived in Section 5 connect $c_{0}(t)$ with $p_{2}(t)$. The air pressure in the surge tank is easily detected continuously by a conventional method. Instead of $p_{2}(t), x(t)$ or $p_{4}(t)$ may be used alternatively. They are again connected with $c_{0}(t)$.

With the air pressure of the surge tank $p_{2}(t)$ detected, the equivalent rate of feeding of alkali-cellulose $q_{0}(t)$ is controlled by the signal $p_{2}(t)$ through a servo. mechanism having transfer function $F(s)$.

When the output of the whole process has to be kept constant, $r(t)=0, \bar{r}$ being the reference value itself. When the transient and frequency responses of the whole process are considered, $r(t)$ should be a step function and a sine wave, respectively. Thus, the block diagram of the whole process is shown in Fig. 7.

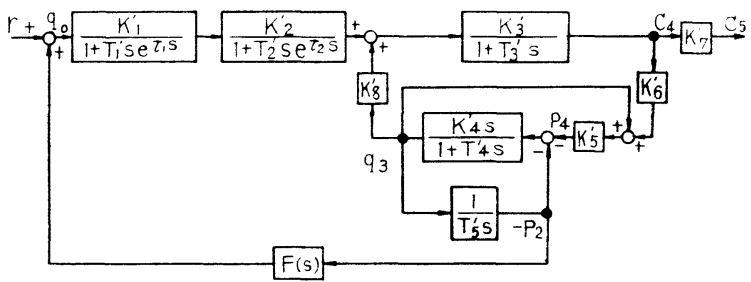

Fig. 7 Block diagram of whole process with rate of cellulose-feeding controlled by surge tank pressure 
The closed loop transfer function of the whole process is derived either by simplifying the block diagram using block diagram algebra or by eliminating the variables other than $R(s)$ and $C_{5}(s)$ from the simultaneous equation consisting of eq. 29 and the following equation :

$$
Q_{0}(s)=R(s)-F(s) \cdot P_{2}(s)
$$

Thus,

$$
\frac{C_{5}(s)}{R(s)}=
$$

$\frac{K_{0}\left(1+T_{4} s\right)}{\left(1+T_{1} s e^{\tau_{1}} s^{s}\right)\left(1+T_{2} s e^{\tau_{2}}\right)\left(1+2 \zeta T_{3} s+T_{3}{ }^{2} s^{2}\right)+K_{2} \cdot F(s)}$ where

$$
K_{2}=k_{6} \bar{p}_{2} / k_{5} k_{7} q_{5} \bar{c}_{4}^{2}\left(\bar{t}_{2}+\gamma \bar{x}\right) .
$$

The characteristic equation of eq. 36 is given by

$$
\left(1+T_{1} s e^{\tau_{1} s}\right)\left(1+T_{2} s e^{\tau_{2} s}\right)\left(1+2 \zeta T_{3} s+T_{3}{ }^{2} s^{2}\right)
$$$$
+K_{2} \cdot F(s)=0 \text {. }
$$
form :

With $\tau_{2}$ ignored, the equation is reduced to this

$$
\begin{gathered}
e^{\tau_{1}} s+\frac{1}{T_{1} s}\left[1+\frac{K_{2}}{\left(1+T_{2} s\right)\left(1+2 \zeta T_{3} s+T_{3}{ }^{2} s^{2}\right)} F(s)\right] \\
=0 .
\end{gathered}
$$

Thus, Satche's method is applicable. For example, if it is possible to select $F(s)$ as

$F(s)=\left(K_{3} / K_{2}\right)\left(T_{1} s\right)\left(1+T_{2} s\right)\left(1+2 \zeta T_{3} s+T_{3}{ }^{2} s^{2}\right), \cdots(40)$ then eq. 41 is rewritten as follows:

$$
e^{\tau_{1}}+\left(\frac{1}{T_{1} s}+K_{3}\right)=0
$$

If $K_{3}>1$, the system becomes absolutely stable. The closed loop transfer function is given by

$$
\frac{C_{5}(s)}{R(s)}=\frac{K_{0}\left(1+T_{4} s\right)}{\left(1+K_{3} T_{1} s+T_{1} s e^{\tau_{1} s}\right)\left(1+T_{2} s\right)\left(1+2 \zeta T_{3} s+T_{3}^{2} s^{2}\right)} .
$$

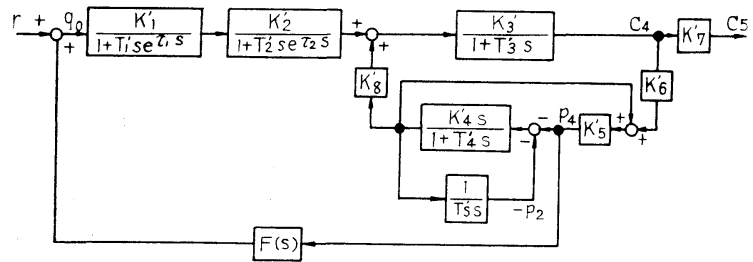

Fig. 8 Block diagram of whole process with rate of cellulose-feeding controlled by surge pressure of slurry press

With the slurry pressure in the reservoir of the slurry press $p_{4}(t)$ detected, instead of $p_{2}(t)$, a block diagram as in Fig. 8 is drawn, and the closed loop transfer function is written as

$$
\begin{aligned}
\frac{C_{5}(s)}{R(s)} & =\frac{K_{0}\left(1+T_{4} s\right)}{\left(1+T_{1} s e^{\tau_{1}}{ }^{s}\right)\left(1+T_{2} s e^{\tau_{2}}{ }^{s}\right)\left(1+2 \zeta T_{3} s+T_{3}{ }^{2} s^{2}\right)} \\
& +K_{4}\left(1+T_{5} s\right) \cdot F(s),
\end{aligned}
$$

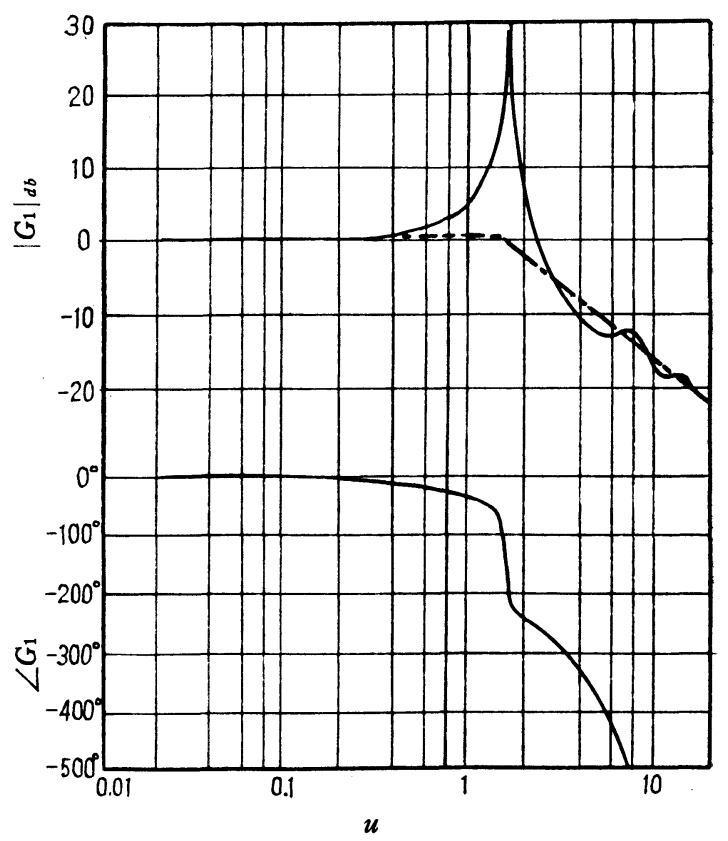

(a) No Control $\left(K_{3}=0\right)$

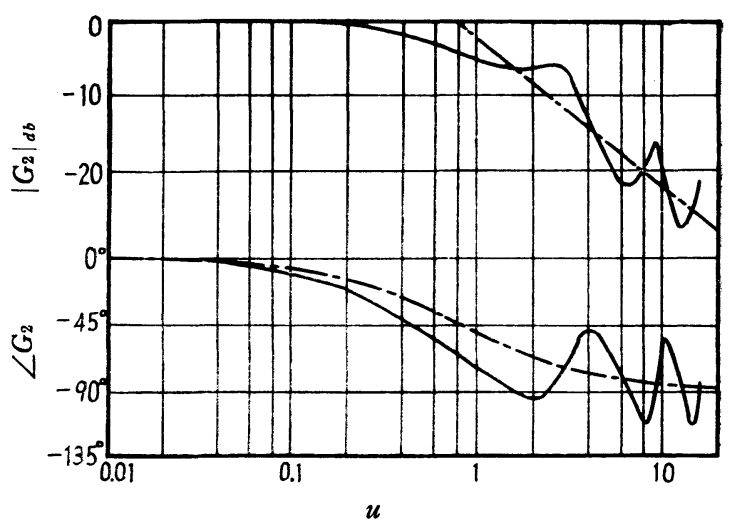

(b) Feedback control $\left(K_{3}=2\right)$

Fig. 9 Effect of feedback control on frequency response of stable $\operatorname{system}(\alpha=2 / 3)$

where

$$
K_{4}=k_{6} / k_{5} k_{7} q_{5} \bar{c}_{4}^{2}, T_{5}=R A \bar{x} /\left(\bar{p}_{2}+\gamma \bar{x}\right) . \cdots \cdots(44)
$$

If $\tau_{2}$ is ignored, the characteristic equation is

$$
e^{\tau_{1} s}+\frac{1}{T_{1} s}\left[1+\frac{K_{4}\left(1+T_{5} s\right)}{\left(1+T_{2} s\right)\left(1+2 \zeta T_{3} s+T_{3}^{2} s^{2}\right)} F(s)\right]=0
$$

If $F(s)$ is selected as

$$
F(s)=\frac{K_{5}}{K_{4}} \frac{T_{1} s\left(1+T_{2} s\right)\left(1+2 \zeta T_{3} s+T_{3}{ }^{2} s^{2}\right)}{1+T_{5} s}, \cdots \cdots(46)
$$

the process becomes stable when $K_{5}>1$. Then, the closed loop transfer function is

$$
\frac{C_{5}(s)}{R(s)}=\frac{K_{0}\left(1+T_{4} s\right)}{\left(1+K_{5} T_{1} s+T_{1} s e^{\tau_{1}} s\right)\left(1+T_{2} s\right)\left(1+2 \zeta T_{3} s+T_{3}{ }^{2} s^{2}\right)} .
$$




\section{Frequency Response of Feedback Control System}

The frequency response of the whole process is altered by the introduction of feedback control. The effect is investigated by numerical calculation comparing the Bode diagrams. Here $\tau_{2}$ is ignored and only eq. 42 is considered.

As eqs. 31 and 42 have a common factor, the functions given below should be compared. Namely:

$$
G_{1}(s)=1 /\left(1+T_{1} s e^{\tau_{1}}\right),
$$

and

$$
G_{2}(s)=1 /\left\{1+T_{1} s\left(K_{3}+e^{i^{s}}\right)\right\} .
$$

Substituting $s=j \omega$ and putting

$$
\alpha=T_{1} / \tau_{1}, u=\tau_{1} \omega
$$

reduce eqs. 48 and 49 to

$$
G_{1}(s)=[(1-\alpha u \sin u)+j \alpha u \cos u]^{-1}, \quad \cdots(51)
$$$$
G_{2}(s)=\left[(1-\alpha u \cos u)+j\left(K_{3}+\alpha u \cos u\right)\right]^{-1} \cdots(52)
$$

The effect of feedback control on frequency response of a stable system is first investigated. Put $\alpha=$ $2 / 3$. The fact that the critical value of $\alpha$ is $2 / \pi$ means

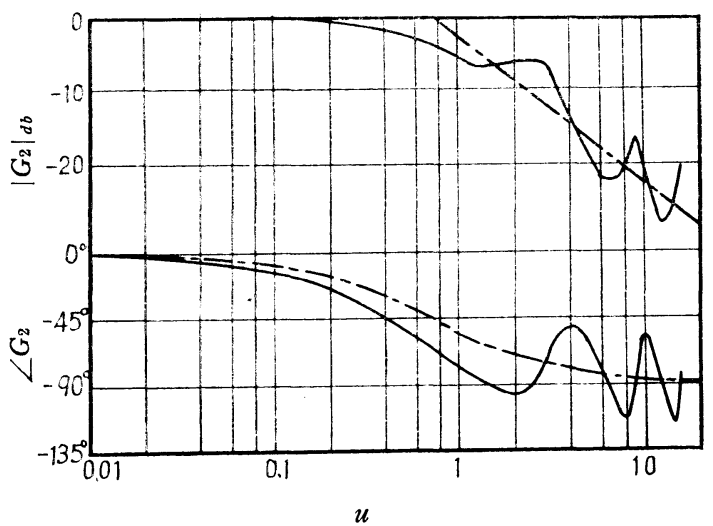

(a) $\boldsymbol{\alpha}=2 / 3$

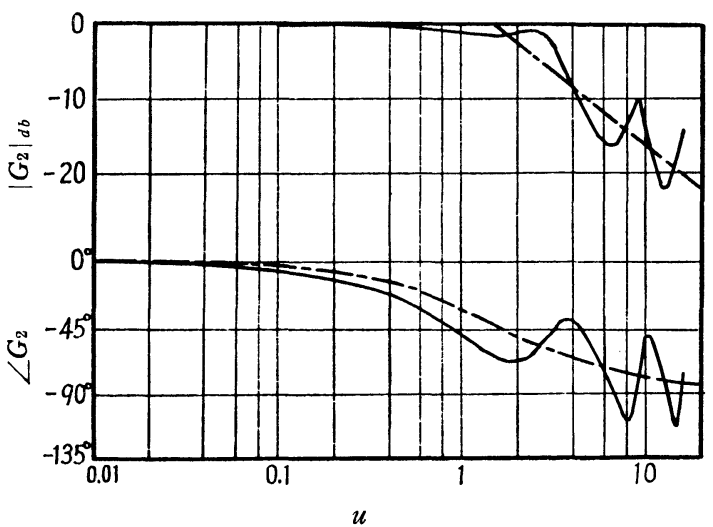

(c) $\alpha=1 / 3$ the stability of the original system. Put $K_{3}=2$, because the condition for absolute stabillity is $K_{3}>1$. The results are illustrated diagramatically in Fig. 9. The chain-line curves are gain and phase asymptotes. Clearly, the sharp peak in the gain plot is eliminated by feedback control. It is a marked improvement in the performance of the whole process.

Now to analyze the effect of feedback control on the frequency response of an unstable system. The value of $K_{3}$ is again selected to be 2 , and the value of $\alpha$ to be 2/3,1/2,1/3 and 1/5. The results of calculation are given in Fig. 10. The peak gain and the corner frequency in the gain plot increase as $\alpha$ increases. The chain-line curves are gain and phase plots of the function $1 /\left(1+T_{1} K_{3} S\right)$.

Before closing the discussion, let us consider the effect of feedback gain $K_{3} \cdot \alpha$ is fixed to be 2, and the Bode diagrams for $K_{3}=1.2,2,4$ and 8 are drawn, which are illustrated in Fig. 11. An increase in $K_{3}$ certainly decreases the break-point frequency and peak gain.

The calculations explained above are only examples.
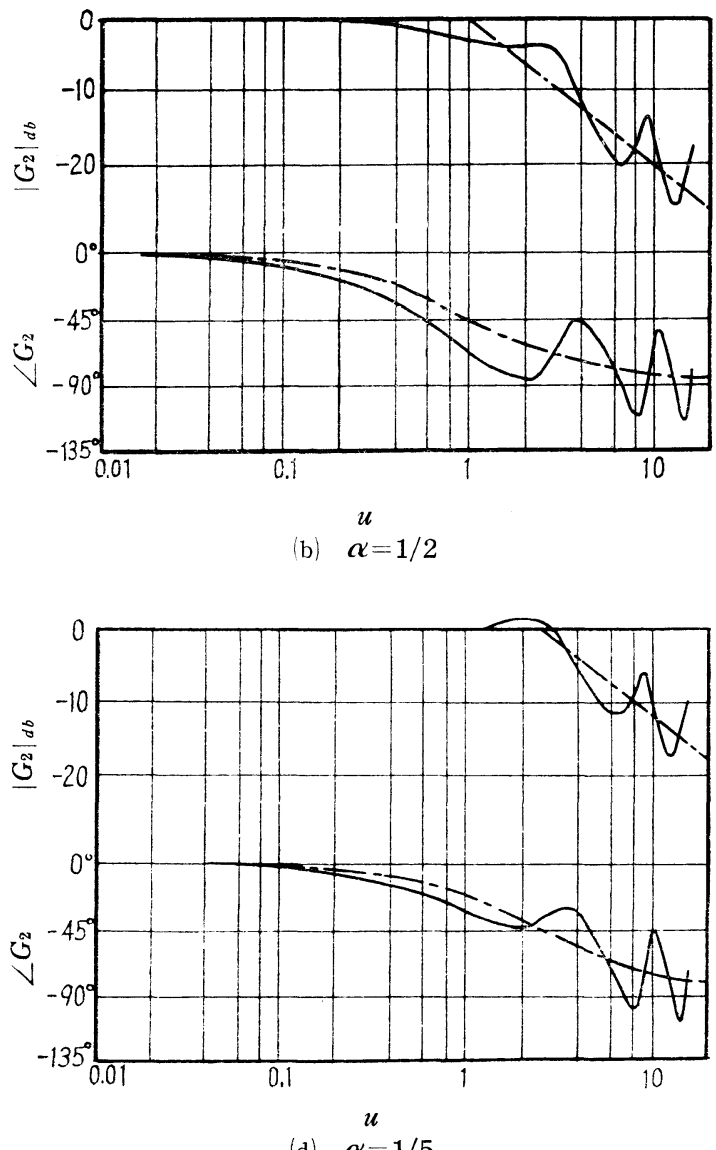

(d) $\alpha=1 / 5$

Fig. 10 Effect of degree of instability of the original system on frequency response of the system with feedback $\operatorname{control}\left(K_{3}=2\right)$ 


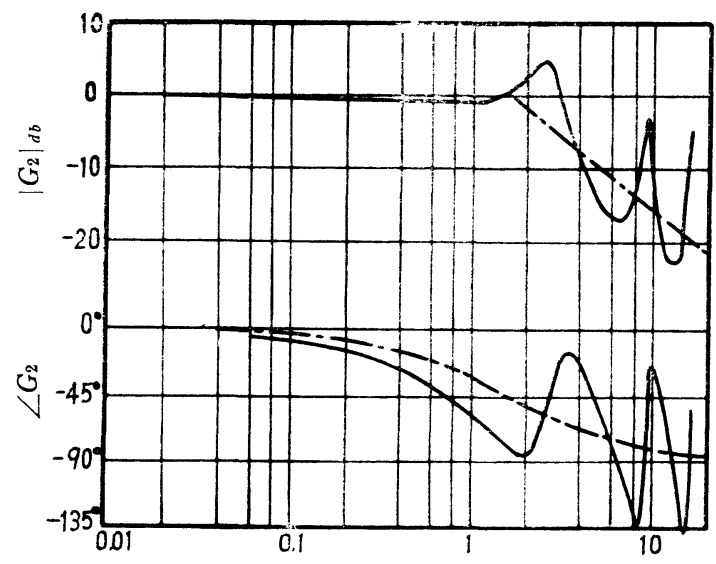

(a) $\stackrel{u}{K_{3}}=1.2$

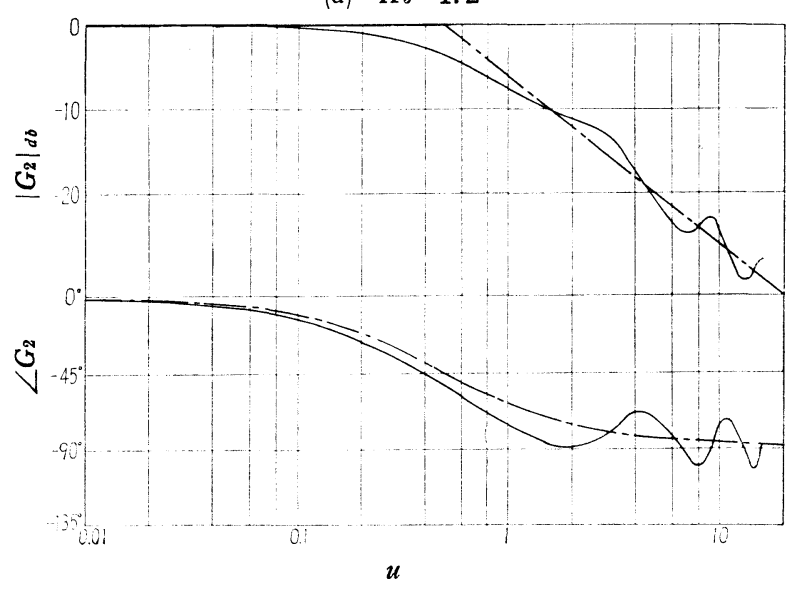

(c) $K_{3}=4$

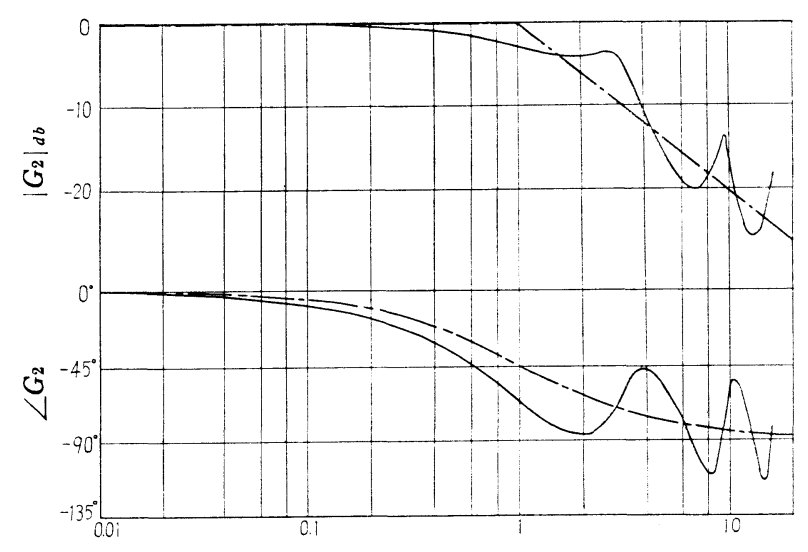

(b) $\stackrel{u}{K_{3}}=2$

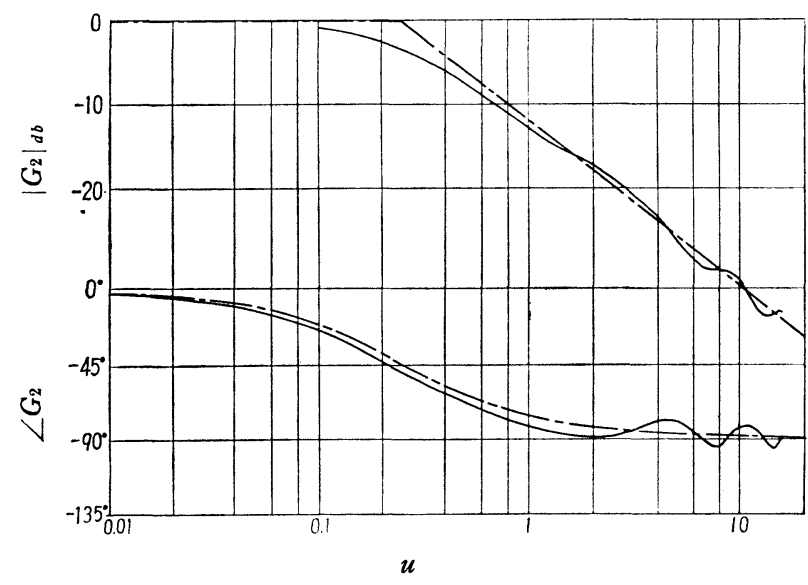

(d) $K_{3}=8$

Fig. 11 Effect of feedback gain on frequency response of unstable system $(\alpha=1 / 2)$

The transfer function of the servo-mechanism is assumed to be expressed by eq. 40 . The feasibility of such a servo-mechanism is never discussed. Nevertheless, these calculations show that stabilization by feedback control improves the performance of the whole process.

\section{Conclusion}

The following conclusions are drawn:

(1) The surge tank has no stabilizing effect if the slurry tank is unstably operated.

(2) The surge tank only slightly improves the frequen. cy response of the process when the slurry tank is stable.

(3) The main role of the surge tank is as a safety device against clogging of the slurry press.

(4) Feed back control of the rate of cellulose feeding is highly effective in stabilizing a slurry tank which is unstable. Either the pressure or level of the surge tank, or pressure of the slurry press reservoir, will do as the control signal.

(5) The feedback control improves the frequency response of the whole process not only when it is unstable but also when it is stable.

\section{Acknowledgement}

The author wishes to thank Dr. Fumio Murakami, Deputy Manager, Technical Department, Toyo Spinning Co., Ltd., for his suggestings and help in the course of the present work.

\section{References}

[1] K. Nakajima : J. Text. Mach, Soc. Jafan, 4, 53 (1958)

[2] M. Satche: J. App. Mech., 16, 419(1949)

[3] H. S. Tsien : J. Amer. Rocket Soc.. 22, 139(1952)

; H. S. Tsien: Engineering Cybernetics Mc-

Graw.Hill, New York, p. 94(1954) 